\title{
Évaluation, concertation, et processus de décision
}

Evaluation, consultation, and the decision-making cycle

Evaluación, concertación y proceso de decisión.

\section{Georges Solaux}

\section{OpenEdition}

\section{Journals}

Édition électronique

URL : https://journals.openedition.org/ries/1893

DOI : 10.4000/ries. 1893

ISSN : 2261-4265

Éditeur

France Education international

Édition imprimée

Date de publication : 1 avril 2003

Pagination : 81-91

ISSN : 1254-4590

\section{Référence électronique}

Georges Solaux, «Évaluation, concertation, et processus de décision », Revue internationale d'éducation de Sèvres [En ligne], 32 I avril 2003, mis en ligne le 01 mars 2008, consulté le 08 juillet 2021. URL : http://journals.openedition.org/ries/1893 ; DOI : https://doi.org/10.4000/ries.1893 


\section{Évaluation, concertation, et processus de décision}

\section{Georges Solaux}

Dans le cadre de cet article, l'analyse des processus de décision de politique éducative sera fondée sur des travaux de recherche essentiellement réalisés sur des projets de réformes concernant l'enseignement du second degré français ${ }^{1}$. De tels projets de réforme comportent des dimensions qui relèvent de la segmentation de l'enseignement en cycles, en séries (générales, technologiques et professionnelles), filières et spécialités, et au sein de ceux-ci, de la répartition des savoirs en disciplines d'enseignement, du partage entre ces disciplines du temps scolaire hebdomadaire et annuel et enfin des modes de certification des connaissances par les examens et diplômes. Il s'agit donc d'un objet de recherche complexe intéressant notamment la définition de la culture scolaire, les modes de sélection des élèves et les professionnalités enseignantes, c'est-àdire en grande partie le «contrôle des modèles selon lesquels la société organise de manière normative ses relations avec son environnement ${ }^{2}$.

La méthode utilisée pour étudier les projets de réforme de l'enseignement secondaire comprend une dimension essentiellement synchronique destinée à appréhender la «sociologie» de la décision politique. Toutefois, la dimension diachronique est utilisée complémentairement, en vue d'apprécier le poids des continuités au regard des ruptures que les uns et les autres semblent introduire dans le système. Il ne s'agit donc pas de développer le fondement juridique du processus de décision mais d'en écrire une sociologie ${ }^{3}$. Afin d'éviter les polémiques et passions susceptibles de se développer sur un thème aussi sensible que la décision politique, nous proposons de ne pas analyser les dix années qui viennent de s'écouler et d'arrêter nos observations au début des années quatre-vingt-dix du siècle dernier. Le constat central de nos travaux tend à présenter le processus de décision comme davantage fondé sur la gestion des rapports de force et les luttes idéologiques que sur une évaluation objective des problèmes posés. Pour le montrer, nous développerons successivement trois points : le cadre théorique mobilisé pour étudier les processus de la décision de politique éducative, le style de management des décisions de politique éducative et les conséquences en matière de processus de décision.

\footnotetext{
1. Nous proposons en bibliographie douze communications traitant de ce sujet.

2. Touraine A., Le retour de l'acteur, Paris, Fayard, 1984.

3. Pour la méthode de recherche voir Solaux G, «Les modalités de changement dans le système éducatif français », Politique et Management Public, volume 20, $\mathrm{n}^{\circ}$ 2, juin 2002, p. 86.
} 


\section{LE CADRE THÉORIQUE}

Peut-on, pour expliquer la décision politique, se limiter à une approche dualiste fondée sur le repérage de régularités causales simples entre des événements clairement identifiables? Ou bien faut-il s'en tenir à une approche pluraliste et compréhensive centrée sur des logiques d'actions d'individus en situation? Peut-on expliquer une réforme en analysant exclusivement du dehors les évolutions voire les reproductions de structures éducatives? Ou bien doit-on s'intéresser uniquement aux logiques d'acteurs? S'enfermer dans de telles alternatives présente le danger de donner du réel une vue qui privilégie soit l'étude de structures sans acteurs, soit l'étude des acteurs indépendamment des structures au sein desquelles ils se meuvent. Il ne nous semble pas possible de limiter notre approche soit à des totalités structurées, soit à des comportements d'acteurs individuels agrégés ou organisés. La réalité sociale des décisions déterminant une réforme du système éducatif peut faire de la place à l'acteur social dans un ensemble structuré donné. L'analyse peut donc intégrer des données macro- et des données micro-sociales. Il en découle que cinq perspectives différentes peuvent être retenues pour analyser le processus de décision.

L'école est segmentée. Chaque filière introduit le jeune dans une hiérarchie qui se subdivise elle-même en autant de paliers hiérarchiques que la filière comprend de cycles. Il en va ainsi de la filière professionnelle qui produit des ouvriers dits qualifiés et munis de titres aussi différents que le CAP, le BEP, le $\mathrm{BPRO}^{4}$. Entrer au lycée professionnel, c'est intégrer la filière ouvrière et, au sein de cette dernière, un certain niveau dans l'excellence des savoirs et savoirfaire reconnus. La hiérarchisation des filières repose sur la hiérarchie des positions sociales auxquelles elles conduisent. La hiérarchie des savoirs généraux, technologiques et professionnels préfigure les hiérarchies professionnelles existantes et peut parfois contribuer à les renforcer. L'introduction du baccalauréat professionnel ne semblant pas avoir bouleversé les hiérarchies scolaires existantes, cette réforme de 1985 ne se réduit-elle pas à remplacer une structure par une autre, voire à perpétuer les clivages existants entre les structures existantes? Si tel était le cas une approche explicative fondée sur le structuralisme génétique et les travaux de Pierre Bourdieu serait pleinement satisfaisante.

A l'opposé de cette approche par les structures et leur reproduction, il est possible d'appliquer aux projets de réformes les concepts relevant davantage de la micro-sociologie et de tenter d'expliquer le processus de décision au moyen des concepts développés par Raymond Boudon dans son analyse du système éducatif du début des années soixante ${ }^{5}$. L'unité de référence ne serait

4. CAP (certificat d'aptitudes professionnelles) et BEP (brevet d'études professionnelles) sont tous deux des diplômes de niveau 5. Le BPRO (baccalauréat professionnel), créé en 1985, est un diplôme de niveau 4 ouvert aux titulaires d'un BEP.

5. Boudon R., L'inégalité des chances, Paris, Colin, 1973. 
plus ici la globalité du système appréhendée par le biais de ses structures constitutives mais l'individu et les groupements d'individus. Les explications des décisions «macro» sont alors à rechercher dans les comportements individuels, dans l'agrégation du «micro». Par exemple, au nombre des variables explicatives de la création du BPRO, il est difficile de décider qui de la demande (agrégation des demandes individuelles de poursuites d'études à l'issue du BEP) ou de l'offre de formation (effet de la structure économique sur l'organisation du système éducatif) a joué le rôle le plus important. Le politique a-t-il décidé de créer le BPRO en vue de faire évoluer l'offre de formation afin de répondre aux besoins économiques, ou bien le fit-il au contraire sous la pression de la demande sociale (agrégation des demandes individuelles) de formation exprimée par les élèves de deuxième année de BEP?

Faut-il d'un autre point de vue envisager le comportement des agents composant le cercle politique proche du ministre de l'Éducation nationale comme une variable explicative de la décision? Ne peut-on pas analyser le processus au moyen du paradigme développé par la sociologie des organisations? Ne peut-on appliquer à l'explication d'une réforme du système éducatif des schèmes d'analyse tels que la centralisation des décisions, l'isolement de chaque catégorie hiérarchique et la pression du groupe sur le décideur, le développement de réseaux de pouvoir parallèles? Les rivalités entre niveaux hiérarchiques, les zones d'incertitude entre domaines de responsabilités sont caractéristiques des grandes organisations administratives et de la dynamique des prises de décision (voir par exemple les tensions qui se sont développées lors de la conception de la "rénovation pédagogique des lycées» en 1990 entre le conseil national des programmes nouvellement créé, l’inspection générale de l'Éducation nationale dont les missions venaient d'être reformulées et la direction des Lycées et Collèges). La décision d'introduire une nouvelle discipline d'enseignement ou d'en réduire une autre implique par ailleurs des coûts qui divisent généralement les tenants du budget et les pédagogues. La raison pédagogique connaît des limites budgétaires et, entre les différents services gestionnaires, existent des rivalités qui traduisent la volonté des uns et des autres d'influer sur la décision en rendant leur point de vue prioritaire et déterminant.

Peut-on, lorsqu'il s'agit d'analyser des décisions de réforme des filières de formation, des horaires et programmes d'enseignement et des modes de certification des connaissances, négliger les apports des sociologues anglosaxons connus sous le nom de "nouvelle sociologie de l'éducation" ou encore de «sociologie du curriculum» ${ }^{6}$ ? Une réforme des formations se traduit généralement par la modification des contenus d'enseignement et de la grille horaire hebdomadaire des enseignements dispensés dans le secteur considéré. L'analyse

6. Forquin JC, École et culture, Bruxelles, De Boeck, 1989. 
comparative des horaires, des épreuves et des coefficients d'examens réalisée sur les différentes sections conduisant aux baccalauréats, avant et après la rénovation pédagogique des lycées, peut sans doute montrer que les choix retenus reposent sur des découpages culturels qui ne sont pas aléatoires.

Enfin, une réforme du système éducatif constitue une expérience de changement dans l'agencement de l'organisation de l'école dont l'origine et l'explication peuvent être trouvées dans les stratégies d'acteurs qui souhaitent utiliser l'incertitude structurelle de toute période de mutation à leur profit, soit pour tenter de renforcer l'existant, soit pour le modifier dans le sens des valeurs et des intérêts qui les animent. Un tel schème d'analyse permettrait de mettre en lumière les conflits sociaux dans le sens développé par Alain Touraine au cours des années soixante-dix ${ }^{7}$. Ainsi, la modification de l'organisation des formations professionnelles initiales ne serait pas seulement une simple adaptation des formations à l'évolution des technologies et de l'organisation du travail, elle serait aussi l'effet d'acteurs qui perçoivent dans l'adoption d'un nouveau système des bénéfices supérieurs aux pertes occasionnées par l'abandon de l'ancien système.

Nous nous trouvons donc placé devant un ensemble de référents théoriques qui, chacun pour ce qui le concerne, semblent présenter quelque utilité dans le développement de l'intelligence de la décision. Compte tenu de la complexité de l'objet d'étude, il semble judicieux de lui appliquer des schémas d'analyse différents mais non exclusifs qui s'articulent autour d'un pivot théorique qui leur soit commun. Nous proposons de retenir comme pivot d'analyse la conception développée par l'action sociale (Touraine) associée au structuralisme génétique (Bourdieu). En ce sens, nous retiendrons l'hypothèse d'un espace social structuré et hiérarchisé, mais ouvert aux stratégies d'acteurs (parmi lesquels il est possible d'identifier des dominants et des dominés) issus de catégories qui entrent en conflit pour s'assurer le contrôle des orientations culturelles en vue de constituer les éléments dominants de l'historicité (et leur permettant de perpétuer leur domination). L'historicité est définie par Touraine comme "la capacité d'une société de construire ses pratiques à partir de modèles culturels et à travers des conflits et des mouvements sociaux ${ }^{8}$. Il est en effet possible d'appliquer à une réforme du système éducatif le concept d'historicité dans la mesure où l'école représente dans nos sociétés l'un des vecteurs essentiels de la transmission culturelle, or «la culture est un enjeu, un ensemble de ressources et de modèles que les acteurs sociaux cherchent à gérer, à contrôler, qu'ils s'approprient ou dont ils négocient entre eux la transformation, en organisation sociale» ${ }^{9}$.

7. Touraine A., Le retour de l'acteur, Paris, Fayard, 1984.

8. Ibid.

9. Ibid. 


\section{LE STYLE DE MANAGEMENT DES DÉCISIONS DE POLITIQUE ÉDUCATIVE}

Le point de vue développé ci-dessus permet de considérer l'État et son représentant qu'est le ministre comme des acteurs parmi d'autres acteurs. Comment concevoir alors leur action? Peut-on affirmer qu'un ministre administre au gré de la pression des groupes d'acteurs? Ne faut-il pas relativiser ce point de vue et penser au contraire que les dirigeants de la société prennent leurs décisions d'abord en fonction d'analyses objectives préalables? N'y a-t-il pas scientifisation du politique? Extension de la consultation scientifique dans les services publics? En d'autres termes, un ministre n'est-il pas d'abord un être rationnel au sens où l'entend Jürgen Habermas? Le politique serait «l'organe d'exécution d'une intelligentsia scientifique qui dégage, en fonction des conditions concrètes, les contraintes objectives émanant des ressources et des techniques disponibles ainsi que des stratégies et des programmes cybernétiques optimaux ${ }^{10}{ }^{10}$. Le technocrate du ministère de l'Éducation nationale serait ainsi capable de prendre les conseils scientifiques les plus avisés et de proposer au ministre les solutions présentant les gages les plus sûrs de réussite dans leur application.

Habermas montre donc qu'il est possible d'inscrire l'action du décideur dans deux types de modèles: soit le modèle "décisionniste», dans lequel subsiste un maximum de marge de liberté à côté d'une pression "technocratique experte» limitée, soit le modèle «technocratique», qui «enferme» davantage la décision dans les progrès de la rationalité de l'expertise que dans la marge de liberté des acteurs (ibid). Mais Habermas ne considère pas que ces deux modèles sont suffisamment représentatifs de la réalité des prises de décision dans la mesure où ils n'intègrent pas ces périodes d'ajustement, de négociations, d'hésitations et de retours en arrière, en un mot tout ce qui caractérise l'action d'un décideur qui, ayant un projet, doit le tordre progressivement, de manière successive et non anticipée, en fonction de l'action et des réactions des acteurs. En ce sens, la décision relève davantage d'une méthode reposant sur ce que les psychologues de l'apprentissage qualifient d' «essais erreurs" que sur l'anticipation et aux deux modèles précédents est préféré un modèle dit "pragmatique»: «ce dernier est une façon de récuser la dichotomie irréductible qu'implique le modèle décisionniste et le monisme réducteur affirmé par le modèle technocratique. Au lieu de cela, le couple décision politique/raison scientifico-technique est engagé dans un processus dynamique d'ajustement réciproque dont Habermas trouve le prototype dans la communication qui s'établit entre les scientific agencies américaines et leurs commanditaires du côté du pouvoir politique. La solution d'un problème passe par un mouvement

10. Habermas J., La technique et la science comme idéologie, Gallimard, 1973. 
d'allées et venues qui aboutit à une détermination progressive du problème luimême en fonction même des solutions qu'il devient au fur et à mesure possible de lui trouver ${ }^{11}$.

L'analyse des processus de décision ne nous a pas permis d'appréhender de réelles pratiques d'évaluation scientifique des problèmes posés avant la fin des années quatre-vingts, pratiques qui semblent se développer davantage aujourd'hui. Le concept d'évaluation a mis plus de temps à émerger en France que dans les pays anglo-saxons. Ce «retard institutionnel» ${ }^{12}$ renvoie à la spécificité des institutions. L'évaluation semble plus développée dans les pays où le principe de transparence prévaut, où la séparation des pouvoirs est très marquée et la capacité de contrôle parlementaire plus forte. Le début des années soixante-dix est marqué en France par la mise en œuvre de la «rationalisation des choix budgétaires» et, progressivement, l'évaluation gagne du terrain dans les pratiques de gestion administrative. A la fin des années quatre-vingts, l'évaluation est présentée comme l'un des quatre axes de la politique de «renouveau du service public» engagée par le gouvernement Rocard. La création du conseil scientifique de l'évaluation en 1990, devenu conseil national de l'évaluation en 1998, traduit dans les faits, et pour l'ensemble du service public, cette volonté politique.

De telles pratiques d'évaluation auraient pu être mises en œuvre dès la fin des années soixante parallèlement au développement de l'économie de l'éducation. Des études prenant en compte les caractéristiques individuelles des élèves, leur origine sociale, leurs niveaux initial et final, les caractéristiques du lycée, celles du corps enseignant, les projets des parents étaient possibles. Elles furent développées par la direction de l'Évaluation et de la Prospective au ministère de l'Éducation nationale au cours des années quatre-vingt-dix. Elles permettent de peser l'impact respectif des diverses variables inventoriées sur le déroulement de la scolarité et, ce faisant, d'aider à construire des réponses adaptées au problème posé. Il ne s'agit pas ici de proposer de fonder les décisions politiques exclusivement sur des évaluations externes, de proposer la mise en place d'une "république d'experts", mais de proposer d'introduire des évaluations externes dans le processus qui conduit à la décision, comme un élément parmi d'autres.

Au niveau du management du processus de décision, deux catégories de ministres peuvent être identifiées dans la période 1970-1990: ceux qui pensent disposer de ce que d'aucuns appellent la «durée» (celle d'une législature par exemple, soit cinq ans) et ceux qui prennent en charge leur département ministériel alors qu'une échéance électorale proche ne leur permet pas d'espérer «durer» au delà de celle-ci (deux ans par exemple).

11. Ibid et lire la préface de Ladmiral J.R.

12. Tenzer N., «L'évaluation: de la modernisation de l'administration à la réforme de l'action publique », Revue française des affaires sociales, Paris, ministère de l’Emploi et de la Solidarité, La Documentation française, pp. 36-40, 2000 . 
À ces deux catégories semblent correspondre des comportements politiques différents. Les premiers se donnent le temps de solliciter l'avis d'experts (mais pas forcément de mobiliser des évaluations à caractère scientifique et externes ${ }^{13}$ ) et utilisent les rapports déposés par ces derniers pour mettre en place une concertation qui réunit tous les acteurs sociaux intéressés par l'école et son organisation. Trois ministres ont procédé de cette manière: Joseph Fontanet, Alain Savary, Lionel Jospin. Par exemple, Lionel Jospin, lors de son intervention au conseil supérieur de l'éducation du 3 décembre 1990, inscrit son action de rénovation du lycée dans le cadre des travaux de la commission Bourdieu-Gros (scientifisation du politique), comme une application logique de la loi d'Orientation sur l'Éducation du 10 juillet 1989 (application juridique) et comme une réponse aux besoins exprimés par le mouvement lycéen d'octobre 1990 (écoute des acteurs) : «le travail que nous allons entreprendre ensemble est en effet l'aboutissement de la dynamique qui a vu le jour avec la mise en place des commissions thématiques (Bourdieu-Gros)... À l'origine de cette réflexion, il y avait, vous vous en souvenez, les "propositions pour l'enseignement de l'avenir" élaborées par le Collège de France... mais si j'ai parlé de dynamique et non de travail d'experts, c'est précisément parce que toute ma démarche a consisté à... faire une véritable réflexion collective. (...) Une méthode progressive et rationnelle nous permettra d'aborder chacune des questions clefs pour l'évolution du lycée de façon beaucoup plus précise et détaillée. Ultérieurement, et une fois vos premières réflexions terminées, j'aurai à arrêter des décisions et à faire des propositions touchant les contenus, les programmes, l'organisation pédagogique du lycée qui seront soumises à votre avis selon la procédure habituelle ${ }^{14}$. La méthode d'élaboration de la rénovation pédagogique des lycées est donc définie et le ministre affirme sa volonté de concertation à toutes les étapes $\mathrm{du}$ processus mis en œuvre.

La seconde catégorie de ministres dispose de peu de temps et ne peut déployer les méthodes de la démocratie consultative avec autant de ferveur. Pressés par le temps, ils doivent prendre rapidement des décisions qui reposent davantage sur ce qu'ils pensent de ce à quoi doit ressembler l'école que sur ce que les autres (experts et acteurs sociaux) pensent de l'organisation de l'école. Il en a ainsi été de Jean-Pierre Chevènement, de René Monory et d'Alain Devaquet.

La distinction effectuée entre ces deux catégories d'acteurs ne doit cependant pas occulter le fait que, dans tous les cas, une concertation a lieu. Dans le premier cas, elle est longue et exhaustive en ce sens que tous les acteurs sont auditionnés en principe sur l'ensemble des sujets. Dans le second cas, la concertation est limitée à des acteurs considérés comme «incontournables» et

13. Externe : qui est réalisée par un expert indépendant des enjeux de la décision à prendre.

14. Conseil Supérieur de l’Education du 3 décembre 1990. 
sur des sujets plus limités. Dans tous les cas l'administration et le ministre disposent de l'opinion d'acteurs. Mais le fait d'avoir entendu une pluralité d'opinions est-il le gage d'une plus grande objectivité ? La prise de décision politique sur la base d'opinions largement sollicitées est-elle plus objectivement et plus scientifiquement fondée que la prise de décisions sur la base de quelques opinions? On peut tout juste dire que, dans le premier cas, le souci démocratique est plus grand que dans le second. Mais, dans les deux cas, les décisions relèvent de convictions voire de fondements idéologiques. En effet chacun des acteurs s'exprime avec ses convictions et à aucun moment il n'est possible de distinguer un interlocuteur qui appuie son argumentation sur le résultat tangible d'une évaluation ou d'une expérimentation.

Même au niveau des rapports d'experts sollicités avant 1990 pour éclairer les décisions au niveau des collèges et des lycées, il est difficile de faire la part entre ce qui relève de la connaissance établie et ce qui appartient au domaine des idéologies. Il nous semble que seul le rapport Legrand, qui a présidé à la rénovation des collèges en 1983, reposait sur une expérimentation préalable (celle des groupe de niveau-matière). D'autres rapports d'experts reposent sur l'association de connaissances développées par les sciences traitant de l'éducation et sur des consultations élargies (rapports Prost et Bourdieu-Gros). Beaucoup ne reposent pas sur des expérimentations préalables : la scientifisation du politique au sens strict du terme évoquée par Habermas ne nous semble pas avoir gagné les coulisses des cabinets ministériels de l'Éducation nationale.

\section{Conséquences en MATIÈre DE PROCESSUS DE DÉCISION}

Le fonctionnement du système éducatif conservant, malgré les rapports d'experts, toute son opacité, il n'est guère possible d'identifier en toute rigueur les variables pertinentes qui agissent effectivement sur l'objet du débat. Ce mode de gouvernement laisse alors la place à l'épanouissement des stratégies d'acteurs et à des prises de décision qui sont d'abord fonction de l'opinion des acteurs dominants. Tout peut être dit puisque rien n'est démontré. Les ministres sont ainsi l'objet de la pression des acteurs sociaux dominants. A partir de ce constat, il n'est pas possible d'affirmer que les «conservateurs » ont plus ou moins de raisons à faire valoir que les "progressistes» et inversement. Ces deux termes recouvrent certes des réalités parfois opposées en matière de conception des politiques éducatives, mais ni les uns ni les autres ne semblent disposer d'arguments objectifs suffisants pour étayer leurs prises de position et pour montrer que la position adverse a tort. Nous proposons donc que conservateurs et progressistes présentent des profils équivalents en matière de fondement des différentes prises de position et que tous deux se définissent d'abord par leurs convictions respectives, peu réalisant des études externes des problèmes pour lesquels ils prétendent disposer de solutions. 
En France par exemple, l'essentiel du débat se situe dans la question qui traverse en permanence le système éducatif depuis le développement du mouvement des compagnons de "l'université nouvelle» à l'issue de la première guerre mondiale et la parution du rapport Langevin-Wallon en 1947. Ce débat est résumé par Raymond Boudon: "l'objectif de l'égalisation des chances fut pris en compte par tous les gouvernements y compris les plus conservateurs. Mais le problème était de déterminer les moyens qui permettraient d'atteindre cet objectif... deux solutions antithétiques se présentent presque immédiatement à l'esprit, et elles peuvent toutes deux s'appuyer sur une argumentation solide. On peut soutenir qu'en mettant les enfants dans un moule scolaire commun aussi longtemps que possible, on se rapprochera du résultat recherché... Mais on peut utiliser une argumentation opposée : en introduisant des filières variées, on permettra à chacun de trouver chaussure à son pied ${ }^{15}$. Ce problème fondamental peut-être dissocié selon les points suivants:

- la définition de la structure pédagogique et du nombre de cycles : ici s'opposent les "unitaires» (défenseurs d'un collège ou d'un lycée à structure identique pour tous reposant sur un nombre limité de séries) et les "pluralistes» (promoteurs d'un collège structuré sur le mode de filières ou d'un lycée organisé sur la base d'un nombre élevé de séries);

- la liberté dans le choix des contenus d'enseignement: dans cette structure de base commune, il faut ensuite définir les marges de liberté individuelle à développer sous forme d'options obligatoires et facultatives;

- la définition de l'espace culturel des lycées par le nombre de disciplines d'enseignement et les horaires hebdomadaires qui sont alloués à chacune d'elles;

- la définition de l'examen terminal du baccalauréat qui constitue d'ailleurs une réponse synthétique à l'ensemble des questions qui précèdent.

D’une manière générale, on peut observer que les réponses progressivement apportées par les différents ministres à ces quatre points se situent sur l'axe de l'ordre et de la continuité beaucoup plus que sur l'axe du changement. En effet, relativement au problème général de l'unité ou de la diversité de l'enseignement du second degré, on peut noter que si les filières dans les collèges ont été supprimées à partir de 1978, la diversité de l'offre et l'inégalité de cette offre, la diversité des publics accueillis selon les collèges perpétuent les filières par le biais des langues vivantes, des options, des établissements euxmêmes. Dans les lycées, le nombre des séries conduisant au baccalauréat a singulièrement diminué depuis 1980. Mais si l'aspect unitaire de l'enseignement secondaire peut paraître se développer, ce phénomène est très nettement atténué par la segmentation en filières générales, technologiques et professionnelles, par le maintien voire le renforcement de la présence d'options obliga-

15. Boudon R., L'idéologie, Paris, Points, collection Essais, 1986, p. 154. 
toires et facultatives ainsi que par la création des enseignements de spécialité qui permettent de personnaliser des parcours de formation, certes plus réduits en nombre, mais plus riches en possibilités de différenciation voire de hiérarchisation et de distinction. Il en va ainsi de l'option facultative "latin» qui continue d'être proportionnellement plus choisie par les élèves de la série scientifique que par les élèves des autres séries. Installés dans la série qui reste la plus noble, ils personnalisent leur profil culturel par l'ajout d'options que les élèves des autres séries choisissent moins fréquemment. Il n’est pas évident dans ces conditions que les «unitaires» l'aient emporté sur les "pluralistes» décrits par Boudon. L'ensemble des données que nous avons rassemblées montre que les évolutions sont pour le moins contrastées et que, sous l'apparence de la rénovation et donc de la nouveauté, se cache un réel conservatisme voire un renforcement des traits dominants de l'organisation antérieure. Cependant, ce conservatisme peut-il être jugé comme négatif par rapport aux innovations que souhaitaient certains ? Rien ne permet de l'affirmer objectivement car aucune étude externe n’a embrassé une problématique aussi complexe.

$\mathrm{Au}$ terme très provisoire de cette réflexion, il semble possible de proposer l'introduction systématique de l'évaluation telle qu'elle est prévue depuis 1998 dans la réforme de l'État, avant la décision (afin de l'instruire) et lors de la mise en place des décisions (afin d'en mesurer les effets). Pour être utile, cette évaluation pourrait rassembler les critères apparemment contradictoire d'externalité au système et de participation du système. Nous proposons une évaluation du problème posé qui soit réalisée par des organismes indépendants des ministères mais qui associeraient à la procédure d'évaluation les acteurs concernés. Il nous semble en effet que si l'objectivité recherchée ne peut être trouvée que dans des expertises externes au système, elle ne peut être utile, c'est à dire assimilée, appropriée par les acteurs, que si ces derniers ont participé et ont été associés à la fois à la définition des variables à évaluer et à l'exploitation des résultats. Ce n'est qu'à ce prix que l'efficacité du dispositif pourrait être renforcée. Il y faut du temps et des modifications d'attitudes, tant chez les évaluateurs qui devraient à cette occasion savoir faire preuve d'écoute tout en demeurant "objectifs», que chez les acteurs sociaux qui devraient quant à eux développer leur culture de l'évaluation et différencier clairement ce qui relève des convictions pré-établies ou des constats objectifs. Il y a dans cette proposition l'avantage de lier l'évaluation à la concertation, ces deux dimensions paraissant à ce jour trop exclusives l'une de l'autre. 


\section{BiblographIE}

BOUDON R. (1986) : L'idéologie, Paris, Points, collection Essais.

TOURAINE A. (1984): Le retour de l'acteur, Fayard.

Revue Pouvoirs (1996). Les groupes d'intérêt, Paris, Seuil.

BOURDIEU P. (1997): Le sens commun, Paris, Minuit..

HABERMAS J. (1973): La technique et la science comme idéologie, Paris, Gallimard.

FORQUIN J.C. (1989): École et culture, Bruxelles, De Boeck.

BRACONNIER S. (1998) : "La technique de l'étude d'impact et le renouveau de l'action publique», Revue du Droit Public, $\mathrm{n}^{\circ} 3$.

LECA J. (66-1993) : «Sur le rôle de la connaissance dans la modernisation de l'État et le statut de l'évaluation ", Revue Française d'Administration publique.

BOURCIER D., KOUBI G. (1999): «Responsabilisation des décideurs et systèmes d'information », l'Actualité Juridique.

Publications de Georges Solaux sur le sujet :

1992, «Rénovation pédagogique des lycées, continuités ou ruptures ?», l'Orientation Scolaire et Professionnelle NE 2, pp. 135-148, coll. André Legrand.

1992, «Du C.A.P et de ses usages, 1959-1992 », Revue Française de Pédagogie NE100 pp 47-58, coll. André Legrand.

1993, «Les décisions de gestion dans l'éducation nationale». Savoir NE 1 pp. 47-68.

1993, «Politiques éducatives et conditions de l'objectif 80 \% d'une classe d'âge au niveau IV », l'Orientation Scolaire et Professionnelle NE 2 pp. 121-145.

1994, L'enseignement professionnel, les CAP, les BEP le baccalauréat professionnel, CNDP-Hachette (coll. Ressources formation).

1995, Le baccalauréat, La Documentation Française Paris.

1995, «Les décisions de politique éducative, le cas du baccalauréat professionnel» dans La construction des politiques éducatives, PUF Paris, ouvrage collectif dirigé par Bernard Charlot et Jacky Beillerot

1999, "L'éducation à l'orientation : les directives ministérielles et l'état de la recherche», L'Orientation Scolaire et Professionnelle, 1999, 28, n²2, pp. 299-325.

2000, L'évaluation des politiques d'éducation (sous la dir. de G. Solaux), CNDP (coll. Documents, actes et rapports), Paris.

2001, Le baccalauréat, éditions le Cavalier bleu, (coll. Idées reçues), Paris. 2000, «L'école et l'égalité des chances» (col. Simon Jacky) dans L'égalité des chances, sous la direction de Koubi G. et Gugliemi G., La Découverte (coll. Recherches), Paris.

2002, «Les modalités de changement dans le système éducatif français», Politique et Management Public, volume 20, n² juin 2002. 